KUNS-2274

YITP-10-47

\title{
Revisiting superparticle spectra in superconformal flavor models
}

\author{
Tatsuo Kobayashian, Yuichiro Nakai ${ }^{b}$ 2, and Ryo Takahashic 3 \\ ${ }^{a}$ Department of Physics, Kyoto University, Kyoto 606-8502, Japan \\ ${ }^{b}$ Yukawa Institute for Theoretical Physics, Kyoto University, Kyoto 606-8502, Japan \\ ${ }^{c}$ Max-Planck-Institut für Kernphysik, Postfach 1039 80, 69029 Heidelberg, Germany
}

\begin{abstract}
We study superparticle spectra in the superconformal flavor scenario with non-universal gaugino masses. The non-universality of gaugino masses can lead to the wino-like or higgsino-like neutralino LSP. Furthermore, it is shown that the parameter space for the higgsino-like LSP includes the region where the fine-tuning problem can be improved. The degeneracy of soft scalar masses does not drastically change when ratios of gaugino masses are of $\mathcal{O}(1)$. The degeneracy of scalar masses for squarks and left-handed sleptons would be good enough to avoid the FCNC problem but that of right-handed slepton masses is weak. However, the overall size of right-handed slepton masses becomes larger when the bino becomes heavier. Thus, that would be favorable to avoid the FCNC problem due to soft scalar masses and A-terms.
\end{abstract}

\footnotetext{
${ }^{1}$ E-mail: kobayash@gauge.scphys.kyoto-u.ac.jp

${ }^{2}$ E-mail: ynakai@yukawa.kyoto-u.ac.jp

${ }^{3}$ E-mail: ryo.takahashi@mpi-hd.mpg.de
} 


\section{Introduction}

Most of free parameters in the standard model appear in the Yukawa couplings leading to quark/lepton masses and mixing angle. It is important to understand what is the origin of hierarchical Yukawa couplings. Indeed, various flavor mechanisms have been proposed to derive the hierarchy of Yukawa couplings.

Supersymmetric extension of the standard model is one of interesting candidates as physics beyond the standard model. Flavor mechanisms leading to hierarchical Yukawa couplings would also affect superpartners of quarks and leptons. Then, we would have a prediction on squark and slepton masses in each flavor mechanism. Such a prediction could be tested when squarks and sleptons are discovered. Furthermore, we have already experimental constraints on squark and slepton masses by flavor changing neutral current (FCNC) processes, even though squarks and sleptons have not been discovered yet [1] Such constraints require a certain degree of degeneracy among squark and slepton masses when those masses are of $\mathcal{O}(100) \mathrm{GeV}$. In addition, A-terms can be another source for the FCNC problem. Then, alignment between Yukawa matrices and A-term matrices is required when squark and slepton masses are of $\mathcal{O}(100) \mathrm{GeV}$.

The flavor mechanism proposed by Nelson and Strassler [4] is one of interesting mechanisms to lead to hierarchical Yukawa couplings. In this flavor scenario, the supersymmetric standard model (SSM) couples with a superconformal (SC) sector between certain energy scales, and it decouples below the energy scale $M_{c}$. Then, superconformal dynamics leads to suppressed Yukawa couplings of quarks and leptons at low energy even if those values are of $\mathcal{O}(1)$ at high energy. (See for early works on this scenario Ref. [5, 6, 7]. Also, new models were proposed recently [8, 9, 10, 11].) Furthermore, superconformal dynamics has another interesting aspect. Suppose that supersymmetry (SUSY) is broken softly at a high energy scale, which is higher than $M_{c}$, and generic values of soft SUSY breaking terms appear in the SSM sector as well as the SC sector. However, superconformal dynamics can suppress squark and slepton masses for any initial values of sfermion masses [12, 5, 6]. Then, small values of squark and slepton masses could be realized at the decoupling energy scale $M_{c}$. Below the decoupling scale $M_{c}$, squark and slepton masses receive usual radiative corrections due to the SSM gaugino masses. Since such radiative corrections are flavor-blind and sizable, we could realize almost degenerate squark and slepton masses at the weak scale. That is, the superconformal dynamics could wash out initial conditions of squark and slepton masses at least for the first and second generations and their degeneracy could be realized at the weak scale. This soft mass spectrum is similar to that in the gaugino mediation [13]. This

\footnotetext{
${ }^{1}$ See also e.g Ref. [2, 3] and references therein.
} 
superconformal flavor mechanism is quite interesting from the viewpoints of both generation of hierarchical Yukawa couplings and dynamical realization of degenerate sfermion masses. We do not need to require a specific SUSY breaking mediation mechanism to avoid the SUSY FCNC problem.

Within this scenario, detailed studies on the low energy spectrum have been done in Ref. [5, 6] by assuming the universal gaugino mass at the energy scale of grand unified theory (GUT), i.e. the GUT relation. Then, it is found that the right-handed sleptons tend to become the lightest superparticle (LSP), unless the gravitino is lighter than them. The slepton LSP is unfavorable from the cosmological viewpoint. In addition, it is found that sfermion masses are expected to be degenerate. However, right-handed slepton masses differ from each other by $\mathcal{O}(10) \%$, that is, their degeneracy is rather weak. For the other sfermion masses, their degeneracy is much better like $\mathcal{O}(1) \%-\mathcal{O}(0.1) \%$. The $U(1)_{Y}$ D-term, $S=\operatorname{Tr} Y m_{i}^{2}$, is helpful both to make the right-handed slepton heavier than the bino and to improve the degeneracy between right-handed slepton masses. However, we may need a sizable value of the $U(1)_{Y}$ D-term. In general, the ratios of A-term matrices to Yukawa matrices can not be controlled by the superconformal dynamics. That may lead to the FCNC problem when squark and slepton masses are of $\mathcal{O}(100) \mathrm{GeV}$.

In this article, we study the superparticle spectra by relaxing the GUT relation among the SSM gaugino masses. Indeed, several mechanisms lead to non-universal gaugino masses [14], e.g. moduli mediation [15], gravity mediation by F-terms of gauge non-singlets [16], anomaly mediation [17], gauge messenger scenario [18], general gauge mediation [19], mirage mediation [20, 21], etc. The reason of the slepton LSP in the above analysis is that radiative corrections due to the bino mass are not large 2 Thus, relaxing the GUT relation, in particular a large bino mass, would be helpful to avoid the slepton LSP. Furthermore, a certain ratio of non-universal gaugino masses is useful to improve/ameliorate the fine-tuning problem [24, 25, 26, 27, 28]. It is also important to study the degeneracy among sfermion masses under the assumption of non-universal gaugino masses. These are our purposes in this paper.

This paper is organized as follows. In section 2, we briefly review the superconformal flavor scenario and give values of sfermion masses at $M_{c}$. In section 3 , by assuming the non-universal gaugino masses, we study the superparticle spectra, in particular, the LSP, and also investigate the fine-tuning of the Higgs sector. In addition, we study the degeneracy among squark and slepton masses. We also discuss about A-terms. Section 4 is devoted to conclusion and discussion.

\footnotetext{
${ }^{2}$ Also, see e.g. [22, 23].
} 


\section{Superconformal flavor scenario}

In this section, we briefly review the superconformal flavor scenario [4], in particular values of squark and slepton masses at the decoupling scale $M_{c}$ [5, 6]. We assume two sectors. One is the SSM sector, which has the gauge group $S U(3) \times S U(2) \times U(1)_{Y}$, three families of matter fields and a pair of Higgs fields, $H_{u}$ and $H_{d}$. It is straightforward to extend it such as GUT groups and more matter/Higgs fields. The other sector is the SC sector including the gauge group $G_{S C}$ and matter fields $\Phi_{r}$. We consider the following superpotential,

$$
W=y^{i j} q_{i} q_{j} H+\lambda^{r s i} \Phi_{r} \Phi_{s} q_{i}+\hat{W}(\Phi)
$$

where $q_{i, j}$ denote quarks and leptons in the SSM and $H$ corresponds to $H_{u}$ and $H_{d}$. Here, the first term denotes the ordinary Yukawa couplings among quarks/leptons and Higgs fields. On the other hand, the second term corresponds to couplings between the SSM matter fields $q_{i}$ and the SC matter fields $\Phi_{r, s}$. Some of SC matter fields must have non-trivial representations under the standard-model gauge group in order to allow these couplings. The third term denotes couplings among only the SC matter fields.

We assume that the superconformal dynamics generates sizable and negative anomalous dimensions of the SC matter fields, $\Phi_{r}$, in the conformal regime between $\Lambda$ and $M_{c}$, where $\Lambda>M_{c}$. Also we assume that the coupling $\lambda^{r s i}$ approaches toward a fixed point. Then, sizable and positive anomalous dimensions $\gamma_{i}$ of the SSM matter fields $q_{i}$ are generated. As a result, the Yukawa couplings $y^{i j}$ at $M_{c}$ are obtained as

$$
y^{i j}\left(M_{c}\right)=y^{i j}(\Lambda)\left(\frac{M_{c}}{\Lambda}\right)^{\left(\gamma_{i}+\gamma_{j}\right) / 2} .
$$

Even if $y^{i j}(\Lambda)=\mathcal{O}(1)$, we would realize suppressed Yukawa couplings $y^{i j}\left(M_{c}\right)$ at $M_{c}$, depend-

ing on values of anomalous dimensions and the length of conformal regime, i.e. $M_{c} / \Lambda$. Thus, this scenario is interesting to generate hierarchical Yukawa couplings among three families. In order to derive the realistic fermion mass hierarchy, one needs different anomalous dimensions for three families, when the length of conformal regime is the same between different families. The third family may not couple to the SC sector in order not to suppress the Yukawa coupling at least for the top quark.

In addition to the above generation of Yukawa hierarchy, the superconformal dynamics has another important aspect. Suppose that the SUSY is broken softly above $\Lambda$ and soft SUSY breaking terms are induced. The SC sector suppresses the gaugino mass of the SC sector, soft scalar masses of $\Phi_{r}$ and squark/slepton masses of $q_{i}$, which couple to the SC sector [12, 5, 6]. However, the superconformal symmetry is not exact, but it is broken by the gauge 
couplings $g_{a}$ and gaugino masses $M_{a}(a=1,2,3)$ in the SSM sector. Then, squark/slepton masses $m_{\tilde{q}_{i}}$ of $q_{i}$ do not continue to suppress, but converge to definite values at $M_{c}$ [5, 6],

$$
m_{\tilde{q}_{i}}^{2} \rightarrow \sum_{a} \frac{C_{i a}}{\Gamma_{i}} \alpha_{a}\left(M_{c}\right) M_{a}^{2}\left(M_{c}\right),
$$

where $\alpha_{a}=g_{a}^{2} /\left(8 \pi^{2}\right)$ and $C_{i a}$ denotes the quadratic Casimir. Here, $\Gamma_{i}$ is a numerical factor of $\mathcal{O}(1)$, which is obtained from the anomalous dimensions, and therefore $\Gamma_{i}$ is flavor-dependent. For concreteness, we show the above convergent values for all squark/slepton masses,

$$
\begin{aligned}
m_{\tilde{Q} i}^{2}\left(M_{c}\right) & =\frac{1}{\Gamma_{Q i}}\left[\frac{16}{3} \alpha_{3} M_{3}^{2}+3 \alpha_{2} M_{2}^{2}+\frac{1}{15} \alpha_{1} M_{1}^{2}\right]\left(M_{c}\right), \\
m_{\tilde{u} i}^{2}\left(M_{c}\right) & =\frac{1}{\Gamma_{u i}}\left[\frac{16}{3} \alpha_{3} M_{3}^{2}+\frac{16}{15} \alpha_{1} M_{1}^{2}\right]\left(M_{c}\right), \\
m_{\tilde{d} i}^{2}\left(M_{c}\right) & =\frac{1}{\Gamma_{d i}}\left[\frac{16}{3} \alpha_{3} M_{3}^{2}+\frac{4}{15} \alpha_{1} M_{1}^{2}\right]\left(M_{c}\right), \\
m_{\tilde{L} i}^{2}\left(M_{c}\right) & =\frac{1}{\Gamma_{L i}}\left[3 \alpha_{2} M_{2}^{2}+\frac{3}{5} \alpha_{1} M_{1}^{2}\right]\left(M_{c}\right), \\
m_{\tilde{e} i}^{2}\left(M_{c}\right) & =\frac{1}{\Gamma_{e i}}\left[\frac{12}{5} \alpha_{1} M_{1}^{2}\right]\left(M_{c}\right),
\end{aligned}
$$

where $Q_{i}, u_{i}, d_{i}, L_{i}$ and $e_{i}$ denote left-handed quarks, right-handed up-sector quarks, righthanded down-sector quarks, left-handed leptons and right-handed leptons, respectively.

The above values of squark/slepton masses squared at $M_{c}$ are smaller by a loop factor $\alpha_{a}$ than $M_{a}^{2}$, although those squarks/slepton masses squared are flavor-dependent. After the decoupling of the SC sector at $M_{c}$, squark/slepton masses squared $m_{q_{i}}^{2}$ receive flavorindependent radiative corrections due to the SSM gaugino masses $M_{a}$ between $M_{c}$ and the weak scale. Such radiative corrections are sizable and of $\mathcal{O}\left(M_{a}^{2}\right)$ when $M_{c}$ is sufficiently higher than the weak scale $M_{Z}$. In this case, the overall size of squark/slepton masses at $M_{Z}$ is determined by radiative corrections between $M_{c}$ and $M_{Z}$.

It is found that the right-handed slepton mass $m_{\tilde{e}}$ is smaller than the bino mass $M_{1}$, i.e.

$$
m_{\tilde{e}}\left(M_{Z}\right)<M_{1}\left(M_{Z}\right)
$$

when $M_{c} \leq 2 \times 10^{16} \mathrm{GeV}$. Then, the right-handed slepton would be the LSP [5, 6] if we assume the GUT relation of gaugino masses,

$$
M_{3}\left(M_{c}\right): M_{2}\left(M_{c}\right): M_{1}\left(M_{c}\right)=\alpha_{3}\left(M_{c}\right): \alpha_{2}\left(M_{c}\right): \alpha_{1}\left(M_{c}\right),
$$

and the gravitino is heavier. That is unfavorable from the cosmological viewpoint.

From the viewpoint of the SUSY flavor problem, it is quite important that squark and slepton masses at $M_{c}$ are suppressed by a loop factor compared with gaugino masses $M_{a}$, 
although those squark and slepton masses are flavor-dependent at $M_{c}$. As said above, flavorindependent radiative corrections between $M_{c}$ and $M_{Z}$ are dominant in the overall size of squark and slepton masses. Hence, squark and slepton masses are almost degenerate. The difference of those masses squared between different families is evaluated as

$$
m_{q_{i}}^{2}\left(M_{Z}\right)-m_{q_{j}}^{2}\left(M_{Z}\right)=m_{q_{i}}^{2}\left(M_{c}\right)-m_{q_{j}}^{2}\left(M_{c}\right)=\left(\frac{1}{\Gamma_{i}}-\frac{1}{\Gamma_{j}}\right) \sum_{a} C_{i a} \alpha_{a}\left(M_{c}\right) M_{a}^{2}\left(M_{c}\right) .
$$

When the GUT relation (6) is assumed, the degeneracy between soft masses is better in the squark sector and there would be no problem in FCNC experiments. The degeneracy is weak in the slepton sector, in particular for the right-handed sleptons. The difference of right-handed slepton masses between different families is of $\mathcal{O}(10) \%$. Such a weak degeneracy may be problematic when the slepton mass is of $\mathcal{O}(100) \mathrm{GeV}$.

The above aspects such as the LSP and mass degeneracy depend on gaugino masses. Indeed, the reason of the slepton LSP is the GUT relation ([6), unless the $U(1)_{Y}$ D-term is sizable. If we assume other values of gaugino mass ratios, we would have different aspects. That is what we will study in the next section.

\section{Non-universal gaugino masses}

Several mechanisms lead to non-universal gaugino masses. Thus, we relax the relation (66) and here we consider the following ratio of gaugino masses,

$$
M_{3}\left(M_{c}\right): M_{2}\left(M_{c}\right): M_{1}\left(M_{c}\right)=\alpha_{3}\left(M_{c}\right): r_{2} \alpha_{2}\left(M_{c}\right): r_{1} \alpha_{1}\left(M_{c}\right),
$$

where $r_{1}$ and $r_{2}$ are real parameters. Each SUSY breaking mediation mechanism would lead to a certain ratio, $\left(r_{1}, r_{2}\right)$. With the above relation (8), we study phenomenological aspects such as the LSP and mass degeneracy by varying the parameters $\left(r_{1}, r_{2}\right)$. In the following analysis, we assume that the $U(1)_{Y}$ D-term is not sizable and neglect it.

\subsection{Superparticle spectrum}

Here, we study the superparticle spectra, in particular the LSP. The right-handed slepton masses receive radiative corrections only from the bino mass. Thus, even if we consider the non-universal relation (8), the right-handed slepton is lighter than the bino for $M_{c} \leq 2 \times 10^{16}$ $\mathrm{GeV}$, i.e. (5). This implies that the bino can not become the LSP, while other superparticles are candidates for the LSP. Thus, only the wino-like or higgsino-like neutralino can be the LSP among neutralinos. We use the following relation,

$$
\mu^{2}\left(M_{Z}\right)=-\frac{M_{Z}^{2}}{2}-\frac{m_{H u}^{2}\left(M_{Z}\right) \tan ^{2} \beta-m_{H d}^{2}\left(M_{Z}\right)}{\tan ^{2} \beta-1},
$$


in order to evaluate the higgsino mass $\mu\left(M_{Z}\right)$. Here, $m_{H u}\left(M_{Z}\right)$ and $m_{H d}\left(M_{Z}\right)$ denote soft scalar masses of the up and down Higgs fields, $H_{u}$ and $H_{d}$, at $M_{Z}$. To evaluate $m_{H u}^{2}\left(M_{Z}\right)$ and $m_{H d}^{2}\left(M_{Z}\right)$, one needs their initial values at $M_{c}$ and stop masses, $m_{\tilde{Q}_{3}}$ and $m_{\tilde{u}_{3}}$ and the Aterm $A_{t}$ corresponding to the top Yukawa coupling, because these as well as gaugino masses $M_{a}$ contribute to radiative corrections of $m_{H u}^{2}\left(M_{Z}\right)$ and $m_{H d}^{2}\left(M_{Z}\right)$. The third family and the Higgs fields do not (strongly) couple to the SC sector in order to lead to the large top Yukawa coupling. Thus, there is no prediction from the superconformal dynamics like Eqs. (3) and (44) for the first and second generations. Here, let us consider the following initial values,

$$
\begin{aligned}
& \text { (i) } \quad m_{Q 3}=m_{u 3}=m_{H u}=m_{H d}=A_{t}=0, \\
& \text { (ii) } \quad m_{Q 3}=m_{u 3}=m_{H u}=m_{H d}=A_{t}=M_{3}, \\
& \text { (iii) } \quad m_{Q 3}=m_{u 3}=m_{H u}=m_{H d}=3 M_{3}, \quad A_{t}=0,
\end{aligned}
$$

at $M_{c}$, for simplicity. Then, by varying $\left(r_{1}, r_{2}\right)$ we can evaluate the superparticle spectra for the cases (i), (ii) and (iii).

For example, we take $M_{3}\left(M_{c}\right)=300 \mathrm{GeV}$ and $\tan \beta=10$. Figures 1 (a)-(c) show the LSP in the case (i) for $M_{c}=2 \times 10^{16}, 10^{14}$, and $10^{12} \mathrm{GeV}$, respectively. Figures 2 (a)-(c) and 3 (a)-(c) are the same figures for the cases (ii) and (iii), respectively.

When we vary $\left(r_{1}, r_{2}\right)$, we can not realize successfully radiative electroweak symmetry breaking $(\mathrm{EWB})$ in a certain region, where the stop becomes tachyonic instead of the Higgs scalar. Such regions with unsuccessful EWB (UEWB) are shown by black triangles in the figures. Around such regions, there is the parameter space corresponding to the squark LSP. The GUT relation, i.e. $\left(r_{1}, r_{2}\right)=(1,1)$, leads to the slepton LSP and there is also the region with small $r_{1}$, which leads to the slepton LSP. The parameter region with large values of both $r_{1}$ and $r_{2}$ leads to the gluino LSP. The regions with (red) " $\times$ " including the region with squares, correspond to the parameter space, where the LSP is wino-like. The regions with (gray) dots including the region with squares, correspond to the parameter space, where the LSP is higgsino-like. Later, we will explain about the region with squares. It is remarkable that there exist broad regions giving the wino- or higgsino-like LSP as long as the value of $r_{2}$ is of $\mathcal{O}(1)$ such as $r_{2} \lesssim 4$. The wino-like neutralino tends to be the LSP around relatively large value of $r_{1}$ such as $r_{1}=\mathcal{O}(30-40)$ with $r_{2}=\mathcal{O}(1)$.

The light higgsino would be favorable to avoid the fine-tuning problem. That is, from the relation (9), it would be natural that $\mu\left(M_{Z}\right)$ is of $\mathcal{O}\left(M_{Z}\right)$. For example, if $\mu\left(M_{Z}\right)$ is of $\mathcal{O}(1) \mathrm{TeV}$ or larger, fine-tuning between $\mu^{2}\left(M_{Z}\right)$ and $m_{H_{u, d}}^{2}\left(M_{Z}\right)$ would be required to lead to the experimental value of $M_{Z}$ by using the relation (9). Following Ref. [29], we introduce 

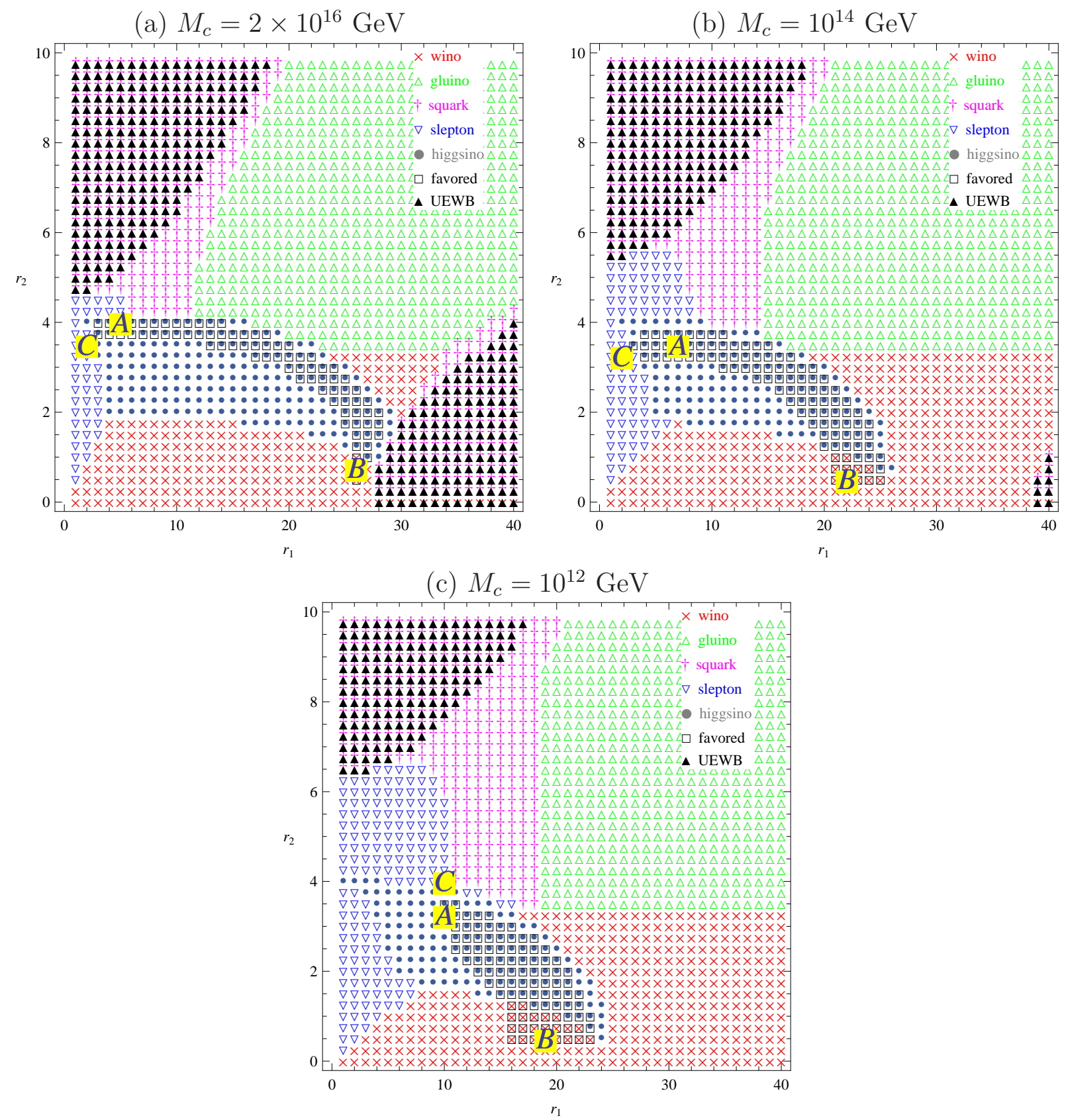

Figure 1: LSP in the case (i): The favored region described by the square means that the fine-tuning problem can be relatively relaxed, $\Delta_{\mu} \lesssim 20$. 

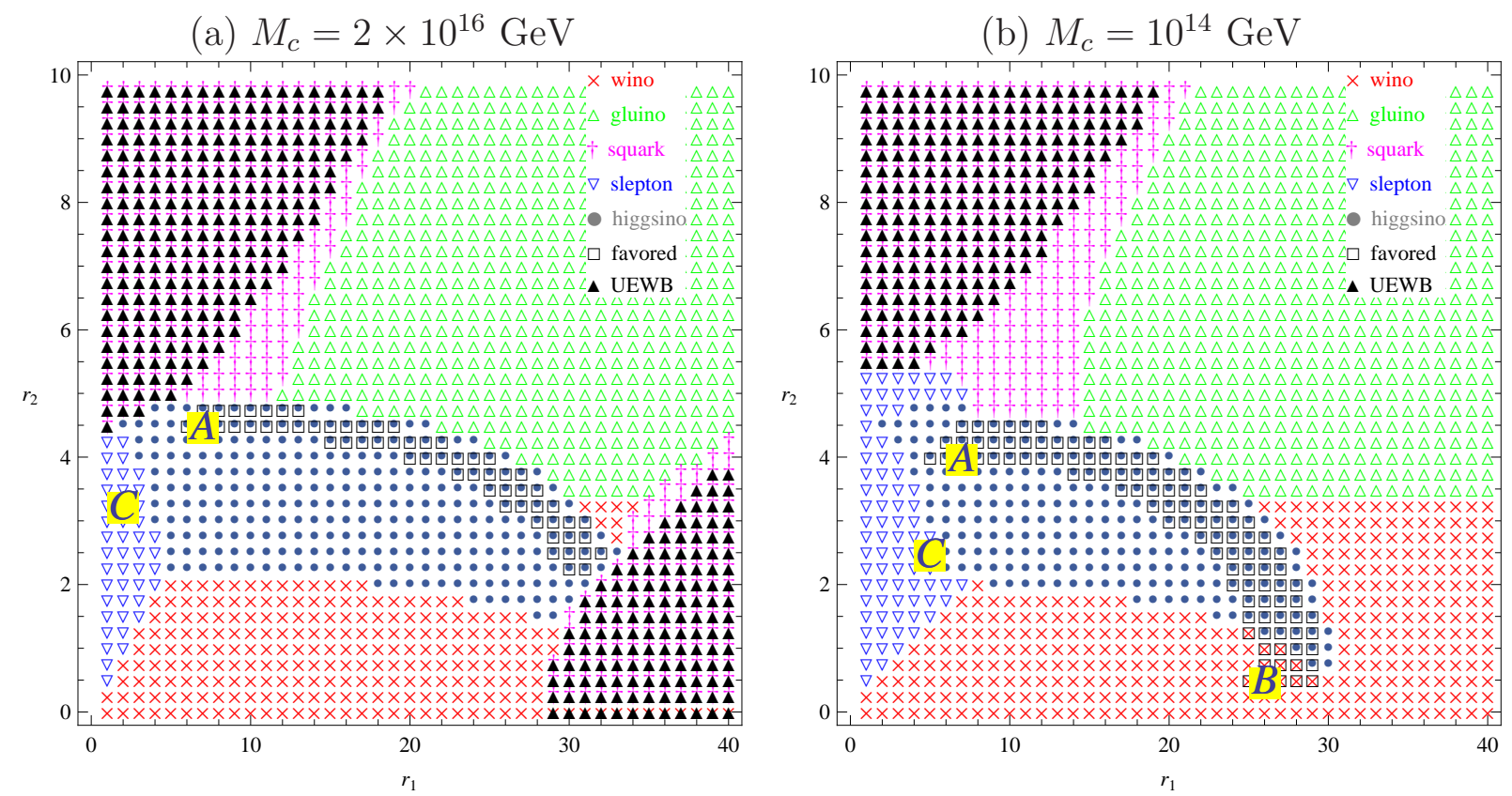

(c) $M_{c}=10^{12} \mathrm{GeV}$

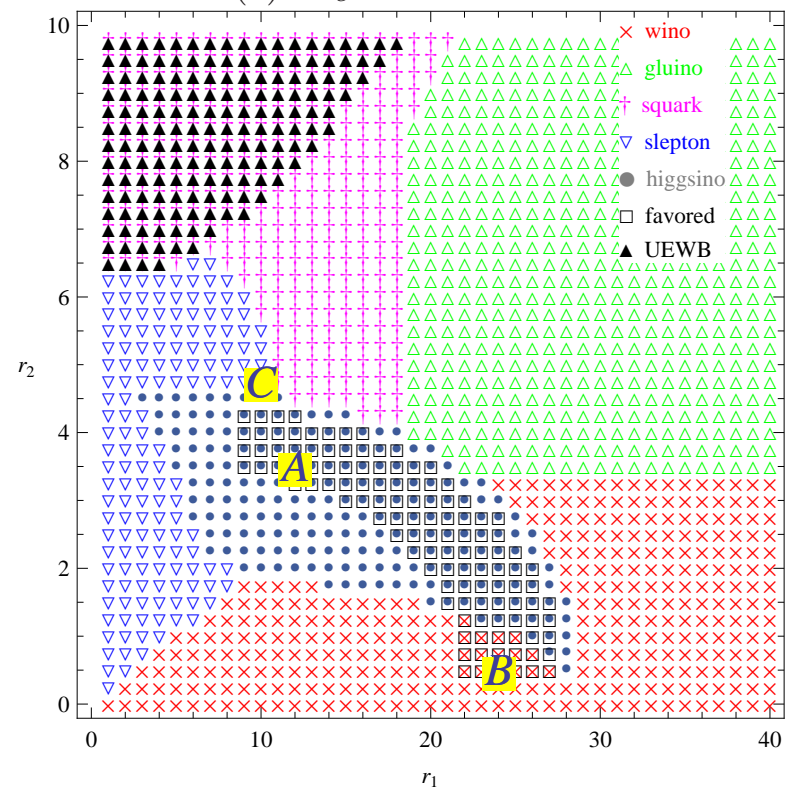

Figure 2: LSP in the case (ii): The favored region described by the square means that the fine-tuning problem can be relatively relaxed, $\Delta_{\mu} \lesssim 20$. 

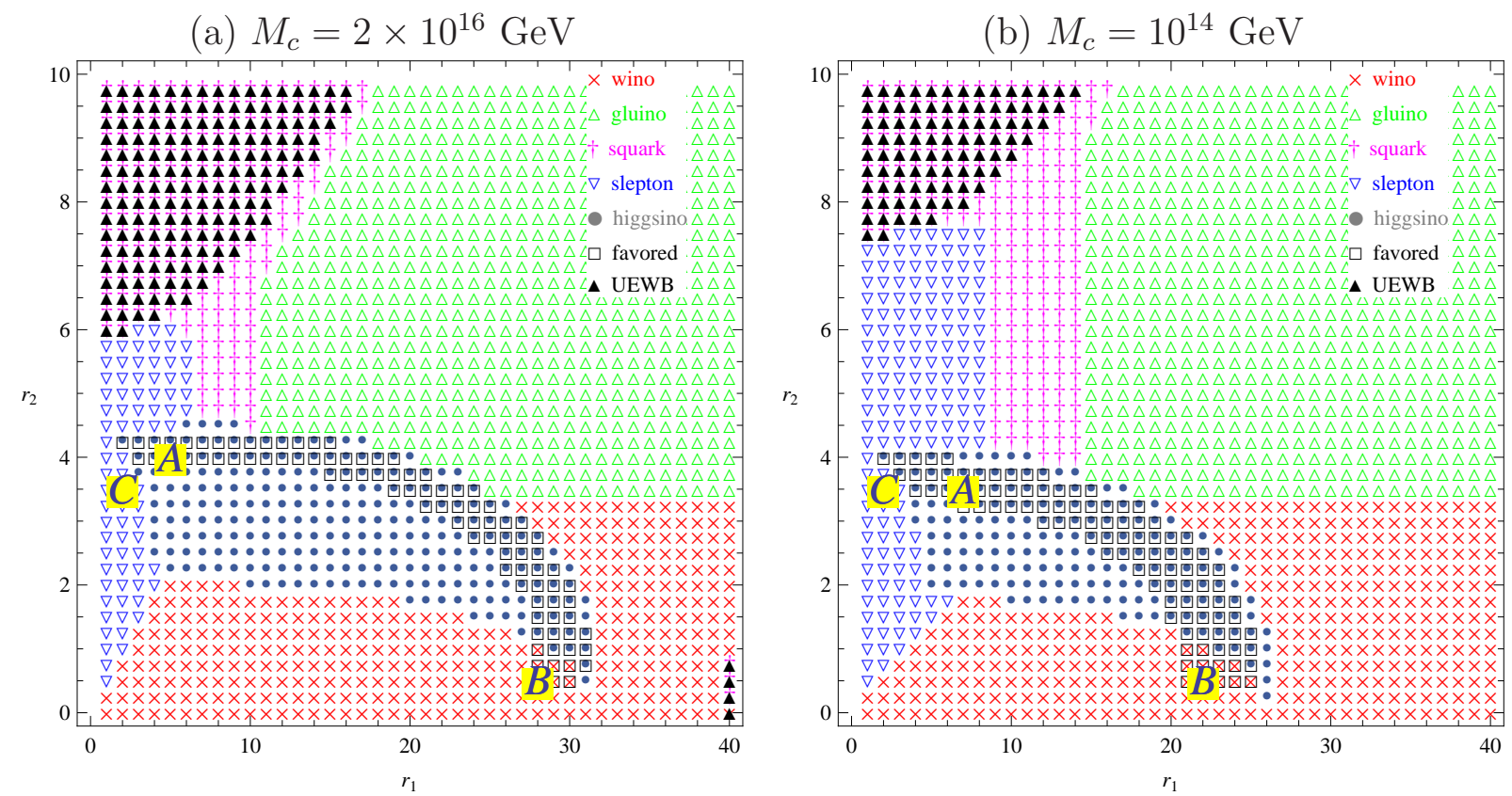

(c) $M_{c}=10^{12} \mathrm{GeV}$

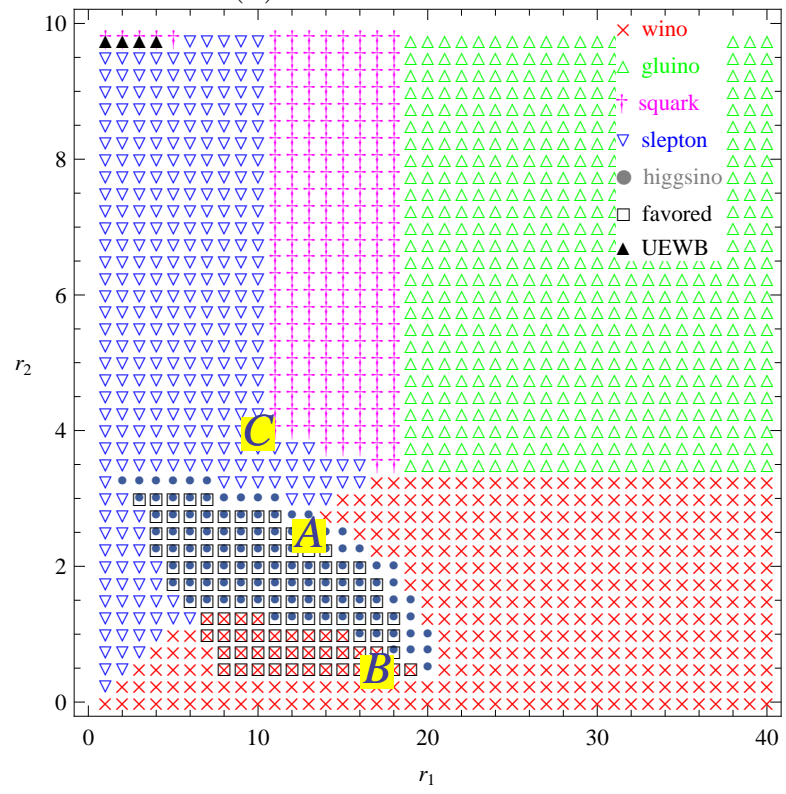

Figure 3: LSP in the case (iii): The favored region described by the square means that the fine-tuning problem can be relatively relaxed, $\Delta_{\mu} \lesssim 20$. 
the fine-tuning index,

$$
\Delta_{\mu}=\frac{1}{2}\left|\frac{\mu}{M_{Z}^{2}} \frac{\partial M_{Z}^{2}}{\partial \mu}\right|=2 \frac{\mu^{2}}{M_{Z}^{2}} .
$$

When $\Delta_{\mu}=20(10)$, the fine-tuning with the degree $5 \%(10 \%)$ is required between $\mu^{2}$ and $m_{H u, H d}^{2}$ in order to lead to the experimental value of $M_{Z}$ in Eq.(9). Then, the conditions for $\Delta_{\mu} \leq 20$ and 10 correspond to $\mu\left(M_{Z}\right) \leq 280$ and $210 \mathrm{GeV}$, respectively. The region with squares in figures corresponds to the parameter space with $\Delta_{\mu} \leq 20$, where we also have $m_{h} \geq 114.4 \mathrm{GeV}$ and superparticle masses larger than experimental lower bounds. We refer such a region as a favored one. Obviously, most of the favored regions correspond to the higgsino-like LSP region. From these figures it is found that as $M_{c}$ increases, the favored region becomes narrow. If a larger value of $M_{3}\left(M_{c}\right)$ such as $500 \mathrm{GeV}$ could be chosen, the favored region also becomes narrow.

Finally, we give examples of soft masses, $\mu$, and the lightest Higgs mass at $M_{Z}$ for cases (i), (ii) and (iii) in Tables 1, 2 and 3. For comparison, the corresponding values in the slepton LSP case (described by (blue) triangles in figures) are also shown in the same tables. The examples corresponding to the higgsino-like, wino-like, and slepton LSP cases are shown by $\mathrm{A}, \mathrm{B}$, and $\mathrm{C}$ in the figures, respectively. It is found that there is not a favored parameter space of the wino-like LSP in the case (ii) with $M_{c}=2 \times 10^{16} \mathrm{GeV}$ because of an unsuccessful EWB. In the wino-like LSP region, typically the value of $r_{1}$ is large, that is, the bino mass is large compared with other gaugino masses. Then, sleptons are heavier than squarks, and slepton masses are of $\mathcal{O}(1) \mathrm{TeV}$. On the other hand, in the higgsino-like LSP region, the value $r_{1}$ is less than those values of $r_{1}$ in the wino-like LSP region. Sleptons are lighter than squarks, but those spectra are compact compared with the case for the GUT relation.

The favored region with squares corresponds to $r_{2} \lesssim 4$ and $r_{1} \lesssim 30$ and such values would be realized in several scenarios. For example, the TeV-scale mirage mediation leads to $\left(r_{1}, r_{2}\right)=(7,3.5)$ [24]. Furthermore, the generalized TeV-scale mirage mediation could lead to larger value of $r_{1}$ [30, 31] such as $r_{1}=\mathcal{O}(10)$. As another example, when the F-term of 75 in $\mathrm{SU}(5) \mathrm{GUT}$ is dominant in the gaugino masses, we have $\left(r_{1}, r_{2}\right)=(5,3)$ [16]. On the other hand, the F-term of $\mathbf{2 4}$ in SU(5) GUT leads to rather smaller values, i.e. $\left(r_{1}, r_{2}\right)=(0.5,1.5)$. The minimal gauge messenger scenario also leads to small values, $\left(r_{1}, r_{2}\right)=(2.5,1.5)$ [18]. Values of $r_{1}$ and $r_{2}$ depend on details of models in the moduli mediation scenario. For example, the so-called O-II model leads to $\left(r_{1}, r_{2}\right)=(29 / 5,3)$ [15, 27]. In the general gauge mediation scenario, we would have naturally non-universal gaugino masses with $r_{1}, r_{2}=\mathcal{O}(1)$, but their concrete values depend on details of models. 


\begin{tabular}{|r||c|c|c|c|c|c|c|c|c|c|}
\hline \multicolumn{1}{|c||}{ LSP } & \multicolumn{2}{|c|}{ higgsino-like $[\mathrm{A}]$} & \multicolumn{3}{c|}{ wino-like [B] } & \multicolumn{3}{c|}{ slepton $[\mathrm{C}]$} \\
\hline$M_{c}[\mathrm{GeV}]$ & $2 \times 10^{16}$ & $10^{14}$ & $10^{12}$ & $2 \times 10^{16}$ & $10^{14}$ & $10^{12}$ & $2 \times 10^{16}$ & $10^{14}$ & $10^{12}$ \\
\hline$r_{1}$ & 5 & 7 & 10 & 26 & 22 & 19 & 2 & 2 & 10 \\
\hline$r_{2}$ & 4 & 3.5 & 3.25 & 0.75 & 0.5 & 0.5 & 3.5 & 3.25 & 4 \\
\hline$M_{1}\left(M_{Z}\right)[\mathrm{GeV}]$ & 617 & 773 & 992 & 3210 & 2430 & 1890 & 247 & 221 & 992 \\
\hline$M_{2}\left(M_{Z}\right)[\mathrm{GeV}]$ & 991 & 776 & 647 & 186 & 111 & 99.7 & 867 & 721 & 797 \\
\hline$M_{3}\left(M_{Z}\right)[\mathrm{GeV}]$ & 865 & 774 & 695 & 865 & 774 & 695 & 865 & 774 & 695 \\
\hline$m_{Q_{1,2}}\left(M_{Z}\right)[\mathrm{GeV}]$ & 1140 & 900 & 744 & 932 & 740 & 623 & 1060 & 869 & 808 \\
\hline$m_{u_{1,2}}\left(M_{Z}\right)[\mathrm{GeV}]$ & 859 & 771 & 708 & 2170 & 1360 & 948 & 780 & 681 & 708 \\
\hline$m_{d_{1,2}}\left(M_{Z}\right)[\mathrm{GeV}]$ & 789 & 699 & 623 & 1270 & 896 & 698 & 769 & 675 & 623 \\
\hline$m_{L_{1,2}}\left(M_{Z}\right)[\mathrm{GeV}]$ & 895 & 655 & 528 & 1530 & 893 & 560 & 749 & 555 & 616 \\
\hline$m_{e_{1,2}}\left(M_{Z}\right)[\mathrm{GeV}]$ & 587 & 565 & 585 & 3050 & 1780 & 1110 & 235 & 162 & 585 \\
\hline$\mu\left(M_{Z}\right)[\mathrm{GeV}]$ & 178 & 228 & 186 & 267 & 237 & 234 & 291 & 274 & 696 \\
\hline$m_{h^{0}}[\mathrm{GeV}]$ & 116 & 116 & 115 & 116 & 118 & 116 & 115 & 114.5 & 115 \\
\hline
\end{tabular}

Table 1: Soft masses, $\mu$, and the lightest Higgs mass at $M_{Z}$ in case (i)

\begin{tabular}{|r||c|c|c|c|c|c|c|c|}
\hline \multicolumn{1}{|c||}{ LSP } & \multicolumn{3}{c|}{ higgsino-like $[\mathrm{A}]$} & \multicolumn{3}{c|}{ wino-like $[\mathrm{B}]$} & \multicolumn{3}{|c|}{ slepton $[\mathrm{C}]$} \\
\hline$M_{c}[\mathrm{GeV}]$ & $2 \times 10^{16}$ & $10^{14}$ & $10^{12}$ & $10^{14}$ & $10^{12}$ & $2 \times 10^{16}$ & $10^{14}$ & $10^{12}$ \\
\hline$r_{1}$ & 7 & 7 & 12 & 26 & 24 & 2 & 5 & 10 \\
\hline$r_{2}$ & 4.5 & 4 & 3.5 & 0.5 & 0.5 & 3.25 & 2.5 & 4.75 \\
\hline$M_{1}\left(M_{Z}\right)[\mathrm{GeV}]$ & 864 & 773 & 1190 & 2870 & 2380 & 247 & 552 & 992 \\
\hline$M_{2}\left(M_{Z}\right)[\mathrm{GeV}]$ & 1110 & 887 & 697 & 111 & 99.7 & 805 & 554 & 946 \\
\hline$M_{3}\left(M_{Z}\right)[\mathrm{GeV}]$ & 865 & 774 & 695 & 774 & 695 & 865 & 774 & 695 \\
\hline$m_{Q_{1,2}}\left(M_{Z}\right)[\mathrm{GeV}]$ & 1230 & 958 & 767 & 763 & 640 & 1030 & 797 & 879 \\
\hline$m_{u_{1,2}}\left(M_{Z}\right)[\mathrm{GeV}]$ & 941 & 771 & 754 & 1550 & 1110 & 780 & 725 & 708 \\
\hline$m_{d_{1,2}}\left(M_{Z}\right)[\mathrm{GeV}]$ & 812 & 699 & 636 & 971 & 754 & 769 & 686 & 623 \\
\hline$m_{L_{1,2}}\left(M_{Z}\right)[\mathrm{GeV}]$ & 1040 & 732 & 590 & 1050 & 705 & 697 & 468 & 707 \\
\hline$m_{e_{1,2}}\left(M_{Z}\right)[\mathrm{GeV}]$ & 822 & 565 & 702 & 2100 & 1400 & 235 & 404 & 585 \\
\hline$\mu\left(M_{Z}\right)[\mathrm{GeV}]$ & 235 & 276 & 254 & 250 & 228 & 433 & 426 & 696 \\
\hline$m_{h 0}[\mathrm{GeV}]$ & 116 & 115 & 115 & 119 & 117 & 116 & 115 & 115 \\
\hline
\end{tabular}

Table 2: Soft masses, $\mu$, and the lightest Higgs mass at $M_{Z}$ in case (ii) 


\begin{tabular}{|r||c|c|c|c|c|c|c|c|c|}
\hline \multicolumn{1}{|c||}{ LSP } & \multicolumn{3}{c|}{ higgsino-like [A] } & \multicolumn{3}{c|}{ wino-like [B] } & \multicolumn{3}{c|}{ slepton [C] } \\
\hline$M_{c}[\mathrm{GeV}]$ & $2 \times 10^{16}$ & $10^{14}$ & $10^{12}$ & $2 \times 10^{16}$ & $10^{14}$ & $10^{12}$ & $2 \times 10^{16}$ & $10^{14}$ & $10^{12}$ \\
\hline$r_{1}$ & 5 & 7 & 13 & 28 & 22 & 17 & 2 & 2 & 10 \\
\hline$r_{2}$ & 4 & 3.5 & 2.5 & 0.5 & 0.5 & 0.5 & 3.5 & 3.5 & 4 \\
\hline$M_{1}\left(M_{Z}\right)[\mathrm{GeV}]$ & 617 & 773 & 1290 & 3460 & 2430 & 1690 & 247 & 221 & 992 \\
\hline$M_{2}\left(M_{Z}\right)[\mathrm{GeV}]$ & 991 & 776 & 498 & 124 & 111 & 99.7 & 867 & 776 & 797 \\
\hline$M_{3}\left(M_{Z}\right)[\mathrm{GeV}]$ & 865 & 774 & 695 & 865 & 774 & 695 & 865 & 774 & 695 \\
\hline$m_{Q_{1,2}}\left(M_{Z}\right)[\mathrm{GeV}]$ & 1140 & 900 & 693 & 947 & 740 & 618 & 1060 & 896 & 808 \\
\hline$m_{u_{1,2}}\left(M_{Z}\right)[\mathrm{GeV}]$ & 859 & 771 & 779 & 2320 & 1360 & 888 & 780 & 681 & 708 \\
\hline$m_{d_{1,2}}\left(M_{Z}\right)[\mathrm{GeV}]$ & 789 & 699 & 643 & 1340 & 896 & 678 & 769 & 675 & 623 \\
\hline$m_{L_{1,2}}\left(M_{Z}\right)[\mathrm{GeV}]$ & 895 & 655 & 509 & 1650 & 893 & 501 & 749 & 596 & 616 \\
\hline$m_{e_{1,2}}\left(M_{Z}\right)[\mathrm{GeV}]$ & 587 & 565 & 760 & 3290 & 1780 & 994 & 235 & 162 & 585 \\
\hline$\mu\left(M_{Z}\right)[\mathrm{GeV}]$ & 263 & 240 & 125 & 265 & 248 & 172 & 349 & 244 & 696 \\
\hline$m_{h^{0}}[\mathrm{GeV}]$ & 121 & 121 & 121 & 124 & 123 & 121 & 121 & 120 & 120 \\
\hline
\end{tabular}

Table 3: Soft masses, $\mu$, and the lightest Higgs mass at $M_{Z}$ in case (iii)

\subsection{Degeneracy}

One of important aspects in the superconformal flavor scenario is that squark and slepton masses between the first and second families are degenerate. It is important to study how much such degeneracy changes by varying $\left(r_{1}, r_{2}\right)$. In this section, we study such an aspect.

As said in the previous section, soft scalar masses squared converge to flavor-dependent values of $\mathcal{O}\left(\alpha_{a} M_{a}^{2}\right)$ at $M_{c}$ as Eqs. (3) and (4). Those values are rather small and flavordependent, and flavor-independent radiative corrections between $M_{c}$ and $M_{Z}$ are dominant in values of soft scalar masses squared at $M_{Z}$. Thus, we estimate the degeneracy degree of soft scalar masses squared by the following values,

$$
\begin{aligned}
\Delta_{\tilde{Q}} & =\Gamma_{Q i} \frac{m_{Q i}^{2}\left(M_{c}\right)}{m_{Q i}^{2}\left(M_{Z}\right)}, & \Delta_{\tilde{u}} & =\Gamma_{u i} \frac{m_{u i}^{2}\left(M_{c}\right)}{m_{u i}^{2}\left(M_{Z}\right)}, \\
\Delta_{\tilde{L}} & =\Gamma_{L i} \frac{m_{L i}^{2}\left(M_{c}\right)}{m_{L i}^{2}\left(M_{Z}\right)}, & \Delta_{\tilde{d}}=\Gamma_{d i} \frac{m_{d i}^{2}\left(M_{c}\right)}{m_{d i}^{2}\left(M_{Z}\right)}, & =\Gamma_{e i} \frac{m_{e i}^{2}\left(M_{c}\right)}{m_{e i}^{2}\left(M_{Z}\right)} .
\end{aligned}
$$

Recall that $m_{Q i}^{2}\left(M_{c}\right)$ is proportional to $1 / \Gamma_{Q i}$ in Eq. (4) and $\Gamma_{Q i}$ is flavor-dependent, where $\Gamma_{Q i}$ would be of $\mathcal{O}(1)$. In Eq. (14), we have put the factors such as $\Gamma_{Q i}$ in the right hand side in order to estimate the degeneracy degree for generic case. Similarly, we have defined $\Delta_{\tilde{u}}$, $\Delta_{\tilde{d}}, \Delta_{\tilde{L}}$ and $\Delta_{\tilde{e}}$. Note that these values depend on only $r_{1}$ and $r_{2}$. Figures 4 (a) $-(\mathrm{c})$ show those values when we vary $r_{1}$ for $r_{2}=2$. We obtain similar behaviors for other values of $r_{2}$ such as $r_{2}=3,4$ or 5 .

First, we comment on the degeneracy of squark masses and left-handed slepton masses except for right-handed slepton masses. For a large value of $r_{1}$ like $r_{1} \gtrsim 20$, the degeneracy 


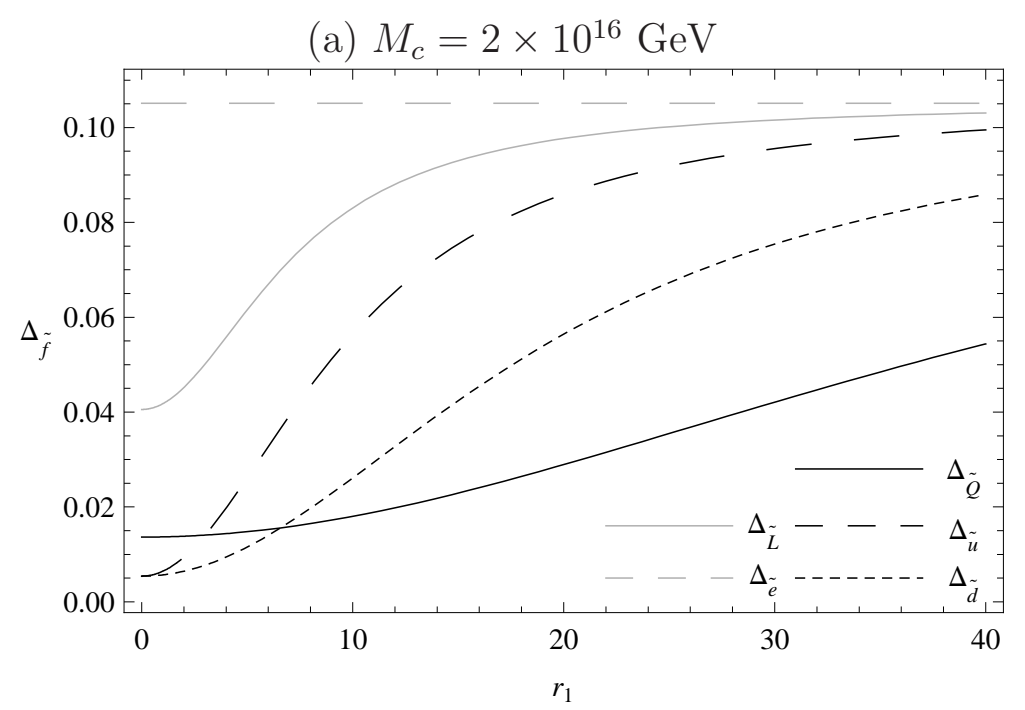

(b) $M_{c}=10^{14} \mathrm{GeV}$

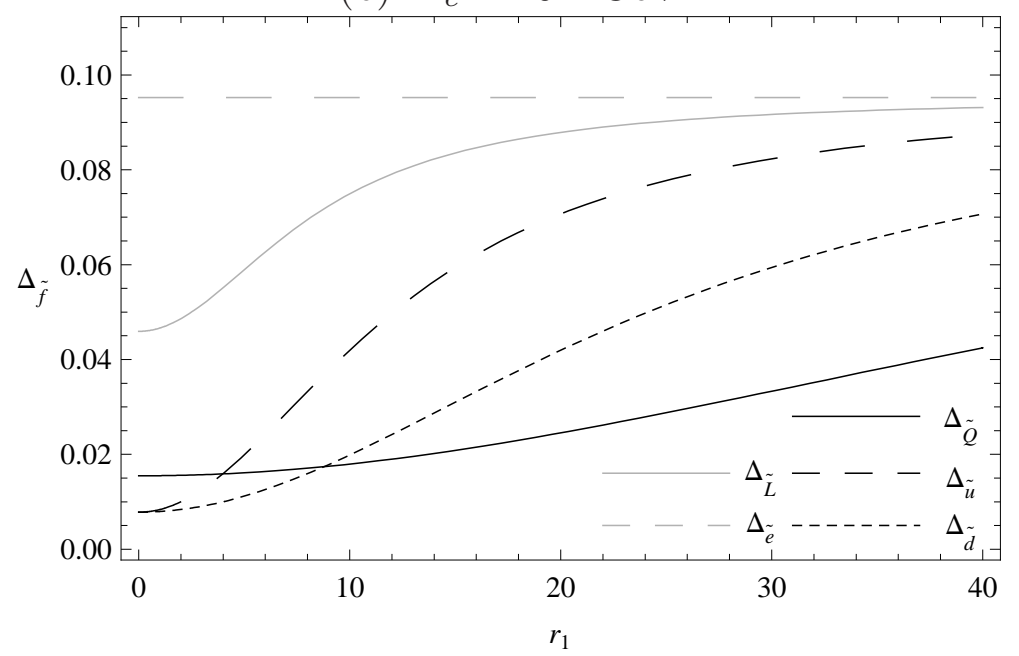

(c) $M_{c}=10^{12} \mathrm{GeV}$

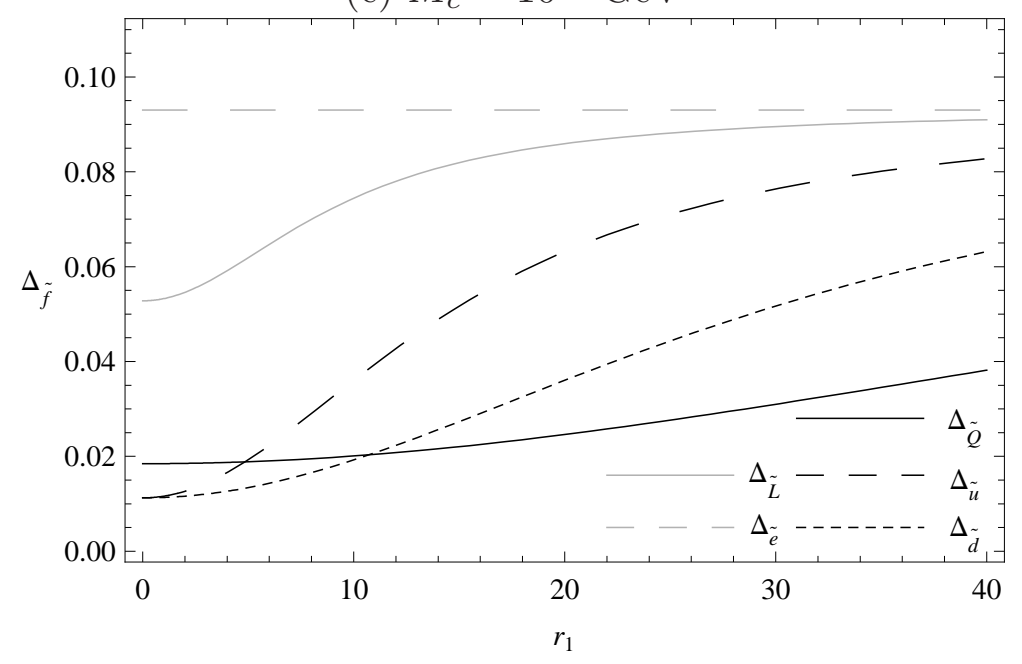

Figure 4: Degeneracy degrees of soft scalar masses squared, $\Delta_{\tilde{f}}$ 
becomes worse. Thus, such a large value of $r_{1}$ would not be favorable from the viewpoint of the FCNC problems. As $r_{1}$ increases, values of $\Delta_{\tilde{Q}}, \Delta_{\tilde{u}}, \Delta_{\tilde{d}}$, and $\Delta_{\tilde{L}}$ approach toward certain values. That is because the bino mass $M_{1}$ becomes dominant in both the numerator and denominator of $\Delta_{\tilde{Q}}, \Delta_{\tilde{u}}, \Delta_{\tilde{d}}$, and $\Delta_{\tilde{L}}$ in the right hand side of Eq. (14). On the other hand, for a small value of $r_{1}=\mathcal{O}(1)$, degeneracies of squark masses and left-handed slepton masses are still good. For squarks, a value such as $r_{1} \lesssim 10-20$ is still favorable. Thus, in these parameter regions, we could avoid the FCNC problem for $\Gamma_{i} \gtrsim 0.1$.

Next we comment on the right-handed slepton masses. The degeneracy for right-handed slepton masses is weak. We may face with the FCNC problem for $\Gamma_{i}=\mathcal{O}(0.1)$. Indeed, the value of $\Delta_{\tilde{e}}$ does not change when we vary $\left(r_{1}, r_{2}\right)$, because both the numerator and denominator of $\Delta_{\tilde{e}}$ in the right hand side of Eq. (14) depend on only the bino mass $M_{1}$. Hence, the degeneracy degree of $\Delta_{\tilde{e}}$ can not be improved by the non-universal gaugino masses (8) and the result on $\Delta_{\tilde{e}}$ is the same as one in Ref. [5, 6]. However, when we increase $r_{1}$ with $M_{3}\left(M_{c}\right)$ fixed, the bino and right-handed sleptons become heavy. Such heavy righthanded sleptons would be helpful to improve the FCNC problem even for weak degeneracy like $\Delta_{\tilde{e}}=\mathcal{O}(0.1)$ [1]. For example, most of the favored parameter space with the higgsino LSP and $\Delta_{\mu} \lesssim 20$, which is plotted by squares, corresponds to $m_{\tilde{e}}>500 \mathrm{GeV}$. In the wino LSP region, we have $m_{\tilde{e}}=\mathcal{O}(1) \mathrm{TeV}$.

We have concentrated on soft scalar masses. In general, A-terms also cause the FCNC problems. Finally, we give a comment on A-terms. Here, we consider the following A-terms

$$
a^{i j} q_{i} q_{j} H
$$

which corresponds to the Yukawa couplings $y^{i j} q_{i} q_{j} H$ in the superpotential (11). Such A-terms are also controlled by the superconformal dynamics. Similar to the Yukawa couplings $y_{i j}$ in (2), they behave as

$$
a^{i j}\left(M_{c}\right) \sim a^{i j}(\Lambda)\left(\frac{M_{c}}{\Lambda}\right)^{\left(\gamma_{i}+\gamma_{j}\right) / 2}
$$

and they are suppressed. However, the ratio $A^{i j}=a^{i j} / y^{i j}$ is important in the FCNC problem, but such a ratio is not suppressed during the conformal regime, because the renormalization group flows of $y^{i j}$ and $a^{i j}$ are the same. In general, flavor-dependent values of $A^{i j}$ are strongly constrained by FCNC experiments. In particular, there is a strong constraint on the $\mu \rightarrow e \gamma$ process. At any rate, the ratio $A^{i j}=a^{i j} / y^{i j}$ can not be controlled by the superconformal dynamics. However, large slepton masses such as $500 \mathrm{GeV}$ or more, which are shown in Tables 1, 2] and 3, are favorable to avoid the FCNC problem through the A-terms [1. 


\section{Conclusion and discussion}

We have studied on superparticle spectra in the superconformal flavor scenario. We have assumed the non-universal gaugino masses. The non-universality in the gaugino masses is quite important. The slepton LSP is not evadable with the GUT relation, unless the $U(1)_{Y}$ D-term is sizable. However, the non-universality of gaugino masses can lead to the winolike or higgsino-like LSP. Furthermore, such a parameter space includes the region, where the fine-tuning between $\mu$ and Higgs soft masses could be improved/ameliorated. The degeneracy of soft scalar masses squared does not change drastically by varying ratios of gaugino masses for the region $r_{1}, r_{2}=\mathcal{O}(1)$. The degeneracy of scalar masses for squarks and left-handed sleptons would be good enough to avoid the FCNC problem, but the degeneracy of righthanded slepton masses is weak. However, the overall size of right-handed slepton masses becomes larger when the bino becomes heavier. That would be favorable to avoid the FCNC problem due to soft scalar masses and A-terms. When $r_{1} \gtrsim 20$, the degeneracy of squark and slepton masses becomes weak.

Our analysis shows that the superconformal flavor scenario with non-universal gaugino masses is quite interesting. Thus, it is intriguing to study model construction leading to realistic Yukawa couplings, although explicit model construction is still a challenging issue. Alternatively, from the AdS/CFT viewpoint, it would be important to study model building in the $5 \mathrm{D}$ warped background 3 .

\section{Acknowledgement}

T. K. is supported in part by the Grant-in-Aid for Scientific Research No. 20540266 from the Ministry of Education, Culture, Sports, Science and Technology of Japan. T. K. and Y. N. are also supported in part by the Grant-in-Aid for the Global COE Program "The Next Generation of Physics, Spun from Universality and Emergence" from the Ministry of Education, Culture, Sports, Science and Technology of Japan. The work of R. T. is supported by the DFG-SFB TR 27. Y. N. is grateful to Institute for Advanced Study for their hospitality where part of this work was done.

\section{References}

[1] F. Gabbiani, E. Gabrielli, A. Masiero and L. Silvestrini, Nucl. Phys. B 477, 321 (1996) arXiv:hep-ph/9604387.

\footnotetext{
${ }^{3}$ See e.g. 32 and references therein.
} 
[2] P. H. Chankowski, O. Lebedev and S. Pokorski, Nucl. Phys. B 717, 190 (2005) arXiv:hep-ph/0502076.

[3] W. Altmannshofer, A. J. Buras, S. Gori, P. Paradisi and D. M. Straub, Nucl. Phys. B 830, 17 (2010) arXiv:0909.1333 [hep-ph]].

[4] A. E. Nelson and M. J. Strassler, JHEP 0009, 030 (2000) arXiv:hep-ph/0006251.

[5] T. Kobayashi and H. Terao, Phys. Rev. D 64, 075003 (2001) arXiv:hep-ph/0103028.

[6] A. E. Nelson and M. J. Strassler, JHEP 0207, 021 (2002) arXiv:hep-ph/0104051.

[7] T. Kobayashi, H. Nakano and H. Terao, Phys. Rev. D 65, 015006 (2002) arXiv:hep-ph/0107030; T. Kobayashi, H. Nakano, T. Noguchi and H. Terao, Phys. Rev. D 66, 095011 (2002) arXiv:hep-ph/0202023; JHEP 0302, 022 (2003) arXiv:hep-ph/0205071; T. Kobayashi, H. Nakano, H. Terao and Y. Yamada, Prog. Theor. Phys. 113, 413 (2005) arXiv:hep-ph/0411288.

[8] D. Poland and D. Simmons-Duffin, arXiv:0910.4585 [hep-ph].

[9] O. Aharony, L. Berdichevsky, M. Berkooz, Y. Hochberg and D. Robles-Llana, Phys. Rev. D 81, 085006 (2010) arXiv:1001.0637 [hep-ph]].

[10] N. Craig, arXiv:1004.4218 [hep-ph].

[11] S. Abel and J. Barnard, arXiv:1005.1668 [hep-ph].

[12] A. Karch, T. Kobayashi, J. Kubo and G. Zoupanos, Phys. Lett. B 441, 235 (1998) arXiv:hep-th/9808178; M. A. Luty and R. Rattazzi, JHEP 9911, 001 (1999) arXiv:hep-th/9908085.

[13] D. E. Kaplan, G. D. Kribs and M. Schmaltz, Phys. Rev. D 62, 035010 (2000) arXiv:hep-ph/9911293; Z. Chacko, M. A. Luty, A. E. Nelson and E. Ponton, JHEP 0001, 003 (2000) arXiv:hep-ph/9911323.

[14] K. Choi and H. P. Nilles, JHEP 0704, 006 (2007) arXiv:hep-ph/0702146.

[15] A. Brignole, L. E. Ibanez and C. Munoz, Nucl. Phys. B 422, 125 (1994) [Erratum-ibid. B 436, 747 (1995)]; T. Kobayashi, D. Suematsu, K. Yamada and Y. Yamagishi, Phys. Lett. B 348, 402 (1995) arXiv:hep-ph/9408322 ; L. E. Ibanez, C. Munoz and S. Rigolin, Nucl. Phys. B 553, 43 (1999) arXiv:hep-ph/9812397]. 
[16] J. R. Ellis, C. Kounnas and D. V. Nanopoulos, Nucl. Phys. B 247, 373 (1984). J. R. Ellis, K. Enqvist, D. V. Nanopoulos and K. Tamvakis, Phys. Lett. B 155, 381 (1985). M. Drees, Phys. Lett. B 158, 409 (1985). G. Anderson, C. H. Chen, J. F. Gunion, J. D. Lykken, T. Moroi and Y. Yamada, arXiv:hep-ph/9609457; K. Huitu, Y. Kawamura, T. Kobayashi and K. Puolamaki, Phys. Rev. D 61, 035001 (2000) arXiv:hep-ph/9903528; S. P. Martin, Phys. Rev. D 79, 095019 (2009) [arXiv:0903.3568 [hep-ph]].

[17] L. Randall and R. Sundrum, Nucl. Phys. B 557, 79 (1999) arXiv:hep-th/9810155; G. F. Giudice, M. A. Luty, H. Murayama and R. Rattazzi, JHEP 9812, 027 (1998) arXiv:hep-ph/9810442.

[18] R. Dermisek, H. D. Kim and I. W. Kim, JHEP 0610, 001 (2006) arXiv:hep-ph/0607169].

[19] P. Meade, N. Seiberg and D. Shih, Prog. Theor. Phys. Suppl. 177, 143 (2009) arXiv:0801.3278 [hep-ph]] ; M. Buican, P. Meade, N. Seiberg and D. Shih, JHEP 0903, 016 (2009) arXiv:0812.3668 [hep-ph]].

[20] K. Choi, A. Falkowski, H. P. Nilles, M. Olechowski and S. Pokorski, JHEP 0411, 076 (2004) hep-th/0411066; K. Choi, A. Falkowski, H. P. Nilles and M. Olechowski, Nucl. Phys. B 718, 113 (2005) hep-th/0503216.

[21] K. Choi, K. S. Jeong and K. i. Okumura, JHEP 0509, 039 (2005) hep-ph/0504037; M. Endo, M. Yamaguchi and K. Yoshioka, Phys. Rev. D 72, 015004 (2005) arXiv:hep-ph/0504036.

[22] S. Komine and M. Yamaguchi, Phys. Rev. D 63, 035005 (2001) arXiv:hep-ph/0007327].

[23] H. Baer, C. Balazs, A. Belyaev, R. Dermisek, A. Mafi and A. Mustafayev, JHEP 0205, 061 (2002) arXiv:hep-ph/0204108.

[24] K. Choi, K. S. Jeong, T. Kobayashi and K. i. Okumura, Phys. Lett. B 633, 355 (2006) hep-ph/0508029; Phys. Rev. D 75, 095012 (2007) arXiv:hep-ph/0612258.

[25] H. Abe, T. Kobayashi and Y. Omura, Phys. Rev. D 76 (2007) 015002 arXiv:hep-ph/0703044.

[26] I. Gogoladze, M. U. Rehman and Q. Shafi, Phys. Rev. D 80, 105002 (2009) arXiv:0907.0728 [hep-ph]].

[27] D. Horton and G. G. Ross, Nucl. Phys. B 830, 221 (2010) [arXiv:0908.0857 [hep-ph]]. 
[28] T. Kobayashi, Y. Nakai and R. Takahashi, JHEP 1001, 003 (2010) arXiv:0910.3477 [hep-ph]].

[29] R. Barbieri and G. F. Giudice, Nucl. Phys. B 306, 63 (1988).

[30] H. Abe, T. Higaki and T. Kobayashi, Phys. Rev. D 73, 046005 (2006) arXiv:hep-th/0511160.

[31] H. Abe, Y. G. Kim, T. Kobayashi and Y. Shimizu, JHEP 0709, 107 (2007) arXiv:0706.4349 [hep-ph]].

[32] K. w. Choi, D. Y. Kim, I. W. Kim and T. Kobayashi, Eur. Phys. J. C 35, 267 (2004) arXiv:hep-ph/0305024. 